\title{
IN VIVO CONDUCTIVITY ESTIMATION USING SOMATOSENSORY EVOKED POTENTIALS AND CORTICAL CONSTRAINT ON THE SOURCE
}

\author{
Sylvain Vallaghé, Maureen Clerc \\ Odyssée Laboratory \\ ENPC - ENS Ulm - INRIA \\ France
}

\author{
Jean-Michel Badier
}

\author{
Laboratoire de Neurophysiologie et Neuropsychologie \\ INSERM, U751 \\ Marseille, France
}

\begin{abstract}
A new method for in vivo conductivity estimation of head tissues is proposed, in the case of a realistic piecewise constant model. Unlike classical electrical impedance tomography methods, for which the conductivity is inferred from a current injection on the scalp, we use an evoked source inside the brain that comes from a somatosensory experiment. The resulting uncertainty with respect to the source is then balanced by strong constraints : we assume the source to be a single dipole located in the cortex, with orientation normal to the cortical surface. Using only EEG data, we are then able to estimate conductivity values, using the MUSIC method to recover the position of the source. Results on simulations show robustness to noise, and the applicability of the method is demonstrated on real data.
\end{abstract}

Index Terms - Electroencephalography (EEG), conductivity estimation, realistic meshes

\section{INTRODUCTION}

Electroencephalography (EEG) is becoming a more and more common functional brain imaging modality. Thus, it is of interest to improve the resolution of the inverse EEG problem, which is spatially poorer than magnetoencephalography (MEG) and functional magnetic resonance imaging (fMRI). The main problem in EEG is its sensitivity to electrical properties of head tissues. In particular, the bad knowledge of skull conductivity can greatly affect the EEG source localization [1]. Further, the skull conductivity value varies highly among subjects. For the purpose of better source estimation, it is hence essential to be able to estimate in vivo conductivities of head tissues.

The main approach taken to achieve conductivity estimation is electrical impedance tomography (EIT), in which a low-intensity current is imposed on the scalp through selected EEG electrodes, and conductivity values are then inferred from potential measurements at the remaining electrodes [2, 3, 4]. One drawback of EIT arises from the insulating effect of the skull, which forces most of the injected current to flow into the scalp, and so lowers the significance of EEG measurements. A different approach is to consider a natural source inside the brain, which allows for more significant measurements of scalp potential. In this case, the electrical source can be controlled by using well-understood stimuli (like median nerve stimulation), combined with MEG for source localization $[4,5,6]$.

In this paper, we present a method that follows the latter approach described above, but differs in our attempt to control the brain source without using MEG source localization, i.e. with only EEG. This is briefly considered in [6], but only on spherical head models and with no constraint on the source, which we demonstrate to have poor robustness to noise with respect to conductivity estimation. Here, our first aim is to achieve a high level of accuracy with the use of realistic meshes and the symmetric boundary element method (BEM) for the forward EEG problem [7]. We also attempt to maintain control of the electrical source position and orientation by constraining it to the cortical surface, and by localizing it with the multiple signal classification (MUSIC) method, which is robust to noise [8]. We present results of the method applied to both simulated and real data.

\section{SYMMETRIC BEM}

\subsection{Forward model for EEG}

Consider an electric potential $V$ which satisfies, inside the head volume $\Omega$ with conductivity $\sigma$, a Poisson equation

$$
\nabla \cdot(\sigma \nabla V)=f \text { in } \Omega
$$

with the following boundary condition on the scalp:

$$
\sigma \partial_{\mathbf{n}} V=j \text { on } \partial \Omega
$$

In an EEG model, the sources inside the brain are represented by $f$. Also, the current on the scalp, $j$, vanishes because the medium around the head (air) is non-conducting.

In this paper we consider a three-layer head model. Conductivities of the brain, the skull and the scalp are respectively 
denoted $\sigma_{1}, \sigma_{2}$ and $\sigma_{3}$. The surfaces enclosing these homogeneous conductivity regions are denoted $S_{1}$ (inner skull boundary), $S_{2}$ (skull-scalp interface) and $S_{3}$ (scalp-air interface).

\subsection{Symmetric BEM formulation}

Careful application of the Green representation theorem to system (1)-(2) leads to a linear relationship through integral operators between the potential $V$ and the normal current $\sigma \partial_{\mathbf{n}} V$ on each of the surfaces ${ }^{1}$, driven by a source term involving the inner current sources $f$ and/or the injected current $j$. More precisely, let $V_{i}$, resp. $p_{i}=\left(\sigma \partial_{\mathbf{n}} V\right)_{i}$ denote the restriction to surface $S_{i}$ of the potential, resp. the normal current. With the formalism presented in [7], the system (1)-(2) for a threelayer head model becomes:

$$
\mathrm{A}_{\sigma}\left[\begin{array}{lllll}
V_{1} & V_{2} & V_{3} & p_{1} & p_{2}
\end{array}\right]^{T}=\mathrm{B}_{\sigma}
$$

where $\mathrm{A}_{\sigma}$ is a symmetric matrix and $\mathrm{B}_{\sigma}$ a vector, described below. The matrix $\mathrm{A}_{\sigma}$ has a block structure

$$
\mathrm{A}_{\sigma}=\left(\begin{array}{cc}
\mathrm{N}_{\sigma} & \mathrm{D}^{T} \\
\mathrm{D} & \mathrm{S}_{\sigma}
\end{array}\right)
$$

with

$$
\begin{gathered}
\mathrm{N}_{\sigma}=\left[\begin{array}{ccc}
\left(\sigma_{1}+\sigma_{2}\right) \mathrm{N}_{11} & -\sigma_{2} \mathrm{~N}_{12} & 0 \\
-\sigma_{2} \mathrm{~N}_{21} & \left(\sigma_{2}+\sigma_{3}\right) \mathrm{N}_{22} & -\sigma_{3} \mathrm{~N}_{23} \\
0 & -\sigma_{3} \mathrm{~N}_{32} & \sigma_{3} \mathrm{~N}_{33}
\end{array}\right] \\
\mathbf{S}_{\sigma}=\left[\begin{array}{cc}
\left(\sigma_{1}^{-1}+\sigma_{2}^{-1}\right) \mathrm{S}_{11} & \sigma_{2}^{-1} \mathrm{~S}_{12} \\
\sigma_{2}^{-1} \mathrm{~S}_{21} & \left(\sigma_{2}^{-1}+\sigma_{3}^{-1}\right) \mathrm{S}_{22}
\end{array}\right] \\
\mathrm{D}=\left[\begin{array}{ccc}
-2 \mathrm{D}_{11} & \mathrm{D}_{12} & 0 \\
\mathrm{D}_{21} & -2 \mathrm{D}_{22} & 0
\end{array}\right]
\end{gathered}
$$

The blocks $\mathrm{N}_{i j}, \mathrm{~S}_{i j}$ and $\mathrm{D}_{i j}$ only depend on the geometric structure of the meshes, and not on the conductivities. The blocks $\mathrm{N}_{i j}$ and $\mathrm{D}_{i j}$ map a potential $V_{j}$ on surface $S_{j}$ to a quantity defined on $S_{i}$. The blocks $S_{i j}$ map a normal current $p_{j}$ on $S_{j}$ to a quantity defined on $S_{i}$.

The vector $\mathrm{B}_{\sigma}$ on the right-hand side of (3) was derived in [7] for the EEG case: supposing $f$ to be supported in the brain compartment, and $j$ in (2) to vanish on the scalp, then

$$
\mathrm{B}_{\sigma}=\left[\begin{array}{lllll}
\left(\sigma_{1} \partial_{\mathbf{n}} v\right)_{1} & 0 & 0 & (v)_{1} & 0
\end{array}\right]^{T}
$$

where $v$ is the solution of $\sigma_{1} \Delta v=f$, i.e. a potential driven by the same source term, but in an infinite domain with conductivity $\sigma_{1}$.

\subsection{Discretization with P1-P0 elements}

The vector of unknowns $\left[\begin{array}{lllll}V_{1} & V_{2} & V_{3} & p_{1} & p_{2}\end{array}\right]^{T}$, once discretized, combines the $P 1$ coefficients of the potential $V$ on all vertices of surface $S_{1}, S_{2}$ and $S_{3}$, and the $P 0$ coefficients of the flux $p=\sigma \partial_{\mathbf{n}} V$ on triangles of surfaces $S_{1}$ and $S_{2}$, as the flux $p$ vanishes on $S_{3}$.

\footnotetext{
${ }^{1}$ Here, $\mathbf{n}$ denotes the outward-pointing normal. Unlike $\partial_{\mathbf{n}} V$, the quantity $\sigma \partial_{\mathbf{n}} V$ is continuous and it is legitimate to consider its restriction to a surface of discontinuity of conductivity.
}

\section{INVERSE SOLUTION}

We model the source by a single dipole. Although it is very simple, it corresponds well to the very focal activity observed in the primary sensory cortex at short latency (before $50 \mathrm{~ms}$ ), in response to a sensory stimulus such as median nerve stimulation.

\subsection{Single dipole localization using MUSIC on the corti- cal surface}

In this section, we present a method for single dipole localization for a fixed conductivity distribution $\sigma=\left(\sigma_{1}, \sigma_{2}, \sigma_{3}\right)$. As in imaging approaches, such as minimum-norm, we use the cortical surface obtained from subject MRI to constrain the localization of the source. Then, in contrast to classical least squares dipole fitting, we search the most probable location of the dipole source within a predefined set of points (the cortical mesh vertices). This allows us to employ scanning methods. Our choice is to use the MUSIC method [8], which gives good results when time series of the sources are independent. Here we assume a single source, and so there cannot be two synchronous sources.

Consider a dipole at a cortical mesh vertex $i$, normal to the cortical surface and with unitary moment. If we solve the system (3) for this dipole and interpolate the values of $V_{3}$ at the electrode positions, we obtain a column vector $g_{\sigma}^{i}$, called the gain field (of this dipole).

Now we consider the EEG measurement data $M$, which is an $n \times m$ matrix, where $n$ is the number of electrodes and $m$ is the number of time samples. Let $M=U S V$ be the singular value decomposition of $M$. For the MUSIC method, we define a signal subspace and a noise subspace. Here, in the case of a single source, the signal subspace is spanned by the first left singular vector in $U$, call it $u_{1}$, and $P=I-u_{1} u_{1}^{T}$ is the orthogonal projector on the noise subspace.

Next we can define a cost function with respect to the cortical mesh vertices :

$$
J(i)=\frac{\left\|P g_{\sigma}^{i}\right\|^{2}}{\left\|g_{\sigma}^{i}\right\|^{2}} .
$$

When $J(i)$ is close to zero, this corresponds to most of the gain field $g_{\sigma}^{i}$ being in the signal subspace, in which case there is a high likelihood for there to be a source located at vertex $i$. In our case of a single dipole, the estimated source position is given by the vertex where $J$ achieves its minimum.

Finally, once we know this vertex and its gain field $g_{\sigma}$, we can compute the amplitude time series $s$ of the dipole using the simple relation $s=\frac{1}{g_{\sigma}^{T} g_{\sigma}} g_{\sigma}^{T} M$, which is the minimum of $\left\|g_{\sigma} s-M\right\|^{2}$.

\subsection{Definition of a cost function for conductivities}

For a given conductivity distribution $\sigma=\left(\sigma_{1}, \sigma_{2}, \sigma_{3}\right)$, we are able to find the most probable location of the dipole source 
within the set of cortical mesh vertices, and we know the corresponding gain field $g_{\sigma}$ and time series $s$. The value of the cost function for this conductivity distribution is then simply defined as

$$
E(\sigma)=\left\|g_{\sigma} s-M\right\|^{2}
$$

This represents the error between the data and the fitted model. The minimum of this cost function corresponds to the parameters of the model (dipole location and conductivity distribution) which give the best fit between simulated and measured scalp potentials. This minimum can be found using an iterative direct search algorithm such as the Nelder-Mead method. At each iteration of the algorithm, a new MUSIC dipole localization is performed.

\section{EXPERIMENTAL RESULTS}

\subsection{Experimental setup}

The data was recorded at Hopital la Timone (Marseille, France), with a 64-channel EEG helmet. An electrical stimulator was positioned on the subject's wrist, and the intensity of the current was chosen according to the sensitivity threshold of the subject, i.e. when the stimulation produced movement of the thumb (around $10 \mathrm{~mA}$ ). The stimulation was repeated at a frequency of $5 \mathrm{~Hz}$ while the potential was sampled at $5 \mathrm{kHz}$. We then averaged the data over about 1000 artifact-free trials to obtain somatosensory evoked potentials (SEP). Two runs were performed, one for the left wrist and one for the right wrist.

\subsection{Head model and system assembly}

A magnetic resonance image of the subject's head was segmented into four surfaces. The ABSOLUt software (Odyssée laboratory, INRIA) was used for the scalp and the cortical surface, and the Brainvisa MEG/EEG toolbox (CNRS UPR 640 - LENA) for inner and outer skull surfaces. The three meshes needed for the BEM (scalp, inner and outer skull) were then decimated using Yams (Gamma laboratory, INRIA) and the final number of vertices per surface was 785 for the inner skull, 842 for the outer skull and 870 for the scalp. For each SEP, we kept only a part of the cortical surface. For instance for the right hand, we kept a region in the left hemisphere that largely included the primary sensory area. The original refinement given by ABSOLUt used about 4000 vertices to compose this cortical region. For a fixed conductivity distribution, the EEG system matrix $\mathrm{A}_{\sigma}$ and the source term $\mathrm{B}_{\sigma}$ were assembled, requiring approximately 10 and 20 minutes, respectively, on a Pentium $\mathrm{PC}$ with $3.8 \mathrm{GHz} \mathrm{CPU}$ and $4 \mathrm{~GB}$ of RAM. Updating these matrices for different $\sigma$ is straightforward given (4) and (5), and so the assembly is performed just once.

\subsection{Simulations and robustness to noise}

To test this method, we produced simulations of EEG data which resemble SEP. We chose a dipole position near the central sulcus, but not exactly on the segmented cortical surface, to take into account the imprecision of the cortex segmentation, as well as the thickness of gray matter. The orientation of the dipole was chosen to be roughly normal to the closest part of cortical surface (figure 1.a). To simulate the short latency response to median nerve stimulation, we took ten samples of a sinusoid around one peak. This corresponds to what can be recorded during $2 \mathrm{~ms}$ at a sampling rate of $5 \mathrm{kHz}$. We then solved the forward problem for this source, for the following dimensionless conductivity values : 1 for brain, $\frac{1}{50}$ for skull, 1 for scalp ${ }^{2}$. Finally we interpolated the values of the scalp potential at 60 electrode positions, and added white Gaussian noise with a SNR of $13 \mathrm{~dB}$, which corresponds to the noise level of evoked potentials.

For the sake of clarity, let us just consider variations of skull conductivity. Without constraint on the source, the position of the estimated dipole using a dipole fit method varies greatly over a range of physiologically relevant skull conductivity values. In figure 1.b, the estimated dipole location is shown for three values of skull conductivity : $\frac{1}{30}$ (yellow), $\frac{1}{43}$ (orange) and $\frac{1}{70}$ (red). The actual dipole is indicated in blue. The depth of the estimated source positions is particularly affected. A plot of the fit error $E$ as a function of the inverse of the skull conductivity value (figure 1.d) shows no clear minimum. This is due to the fact that the dipole position has much more weight on the fit error than the conductivity.

With cortical constraint, the dipole is always localized at the same position for all skull conductivity values (figure 1.c, note that the MUSIC map is the inverse of $J$ ). As a result, the cost function $E$ strongly depends on the conductivities. The plot of $E$ has a well-defined shape (figure 1.e). The minimum is achieved for a skull conductivity of about $\frac{1}{43}$, and corresponds to a relative error of $15 \%$. This demonstrates the interest of the method : with the estimated skull conductivity value, the dipole fit method gives a good estimation (1.b, orange point) of the actual position (1.b, blue point). The distance between the two is $2.8 \mathrm{~mm}$.

\subsection{Real data}

In figures 2.a and 2.b, we show the normalized fit error $E$ as a function of the inverse of the skull conductivity value (for brain and scalp conductivities fixed to 1), for both right hand and left hand SEP. In both cases, the fit error has a welldefined minimum. We obtained the following skull conductivity values when searching with the Nelder-Mead method : 0.0112 for the right hand and 0.0123 for the left hand. The relative difference between the two is $9 \%$. These values are in

\footnotetext{
${ }^{2}$ Conductivity values are normalized with respect to brain conductivity, which is then equal to 1 .
} 


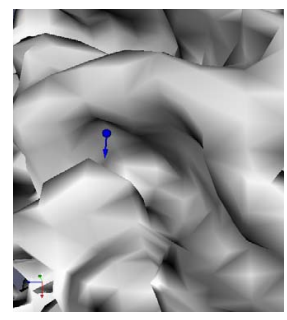

(a) Dipole position.

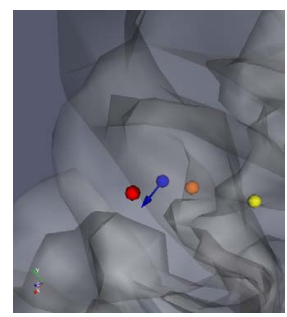

(b) Dipole fits.

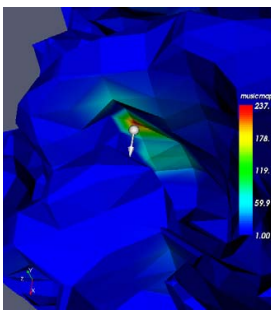

(c) MUSIC map.

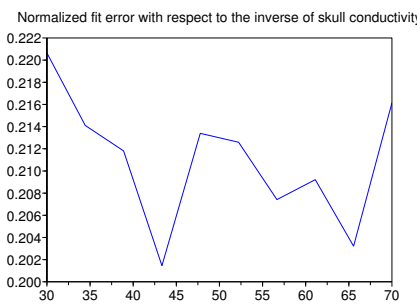

(d) No cortical constraint.

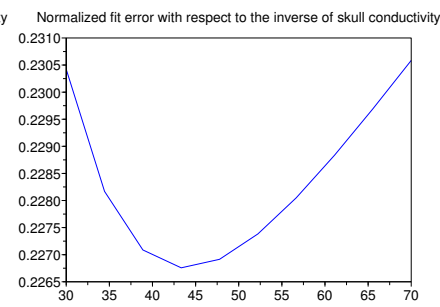

(e) Cortical constraint.
Fig. 1. Simulations.

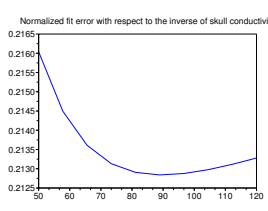

(a) Right hand.

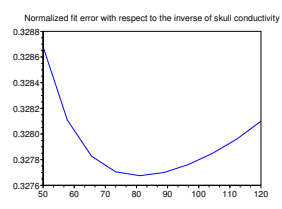

(b) Left hand.

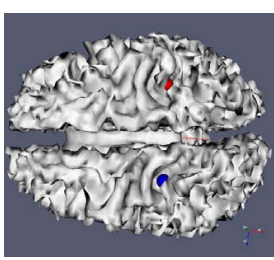

(c) Dipole positions.
Fig. 2. Real data from SEP experiment.

the same range as the results of other real experiments $[3,4,5$, $6]$, and the rather small difference between left and right hand suggests that the results are indeed reproducible. Figure 2.c shows the positions of the dipoles estimated by the method : blue is for the right hand stimulus and red is for the left hand stimulus.

\section{CONCLUSION}

We have presented a new approach for conductivity estimation, using only an evoked source and EEG. We showed that in the presence of noise, it is hard to estimate both one single dipole and the conductivity distribution without adding constraints. We localized the source by scanning the vertices of a cortical surface mesh, and used the MUSIC method to reduce sensitivity to noise. With this constraint, the variations of the model-data fit error depends much more on conductivity values than on the dipole position, which makes conductivity estimation possible in presence of noise. On real data, the results were consistent between right and left hand stimulus, and were in agreement with previous literature.

\section{ACKNOWLEDGMENTS}

The authors would like to thank Patrick Marquis and Christian Bénar of INSERM U751 for their help during the data acquisition procedure. This work is partially supported by the INRIA COLOR EEG++ Grant (INRIA - La Timone hospital, Marseille), and the French Research Ministry Large Data Set ACI Obs-Cerv.

\section{REFERENCES}

[1] G. Huiskamp, M. Vroeijenstijn, R. van Dijk, G. Wieneke, and A. C. van Huffelen, "The need for correct realistic geometry in the inverse EEG problem," IEEE Trans. on Biomed. Engin., vol. 46, no. 11, pp. 1281-1287, Nov. 1999.

[2] T.C. Ferree, K.J. Eriksen, and D.M. Tucker, "Regional head tissue conductivity estimation for improved EEG analysis," IEEE Transactions on Biomedical Engineering, vol. 47, no. 12, pp. 1584-1592, Dec. 2000.

[3] T.F. Oostendorp, J. Delbeke, and D.F. Stegeman, "The conductivity of the human skull: Results of in vivo and in vitro measurements," IEEE Transactions on Biomedical Engineering, vol. 47, no. 11, pp. 1487-1492, Dec. 2000.

[4] S. Gonçalves, J.C. de Munck, J.P. Verbunt, R.M. Heethaar, and F.H. Lopes da Silva, "In vivo measurement of the brain and skull resistivities using an EIT-based method and the combined analysis of SEF/SEP data.," IEEE Transactions on Biomedical Engineering, vol. 50, no. 9, pp. 1124-8, Sept. 2003.

[5] U. Baysal and J. Haueisen, "Use of a priori information in estimating tissue resistivities - application to human data in vivo," Physiol. Meas., vol. 25, pp. 737-748, 2004.

[6] David Guttiérrez, Arye Nehorai, and Carlos H. Muravchik, "Estimating brain conductivities and dipole source signals with EEG arrays," IEEE Transactions on Biomedical Engineering, vol. 51, no. 12, pp. 2113-2122, Dec. 2004.

[7] J. Kybic, M. Clerc, T. Abboud, O. Faugeras, R. Keriven, and T. Papadopoulo, "A common formalism for the integral formulations of the forward EEG problem," IEEE Transactions on Medical Imaging, vol. 24, no. 1, pp. 1228, Jan. 2005.

[8] John C. Mosher, Paul S. Lewis, and Richard M. Leahy, "Multiple dipole modeling and localization from spatiotemporal MEG data," IEEE Transactions on Biomedical Engineering, vol. 39, no. 6, pp. 541-553, 1992. 